\title{
Mixed methods analysis of Health-Related Quality of Life in ambulant individuals affected with RYR1-related myopathies pre-post- $\mathrm{N}$-acetylcysteine therapy
}

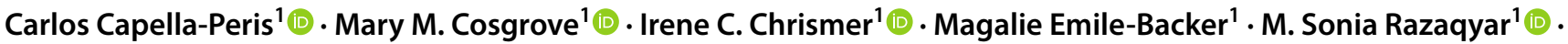 \\ Jeffrey S. Elliott ${ }^{1} \cdot$ Anna Kuo $^{1} \cdot$ Paul G. Wakim ${ }^{2}$ (1) $\cdot$ Katherine G. Meilleur ${ }^{1}(\mathbb{C}$
}

Accepted: 11 January 2020 / Published online: 10 February 2020

(c) Springer Nature Switzerland AG 2020, corrected publication 2020

\begin{abstract}
Purpose To characterize Health-Related Quality of Life (HRQoL) in ambulant individuals with RYRI-RM and to determine if a qualitative PRO tool (subjective self-assessment) complements PROMIS and Neuro-QoL scales to detect changes in HRQoL in ambulant individuals with $R Y R I$-RM post $N$-acetylcysteine (NAC) treatment.

Methods The study used a mixed methods research (MMR) design applying methodological triangulation. Qualitative data were collected via semi-structured interviews using open-ended questions. Quantitative data were gathered through PROMIS and Neuro-QoL instruments. Additionally, qualitative data were transformed into quantitative data for subjective self-assessment and frequency analyses.

Results Qualitative results identified five domains and 33 subdomains as areas of interest. The most valuable were the importance of social impacts, the development of several coping strategies, both physical and psychological, and the identification of fatigue and weakness as key symptoms. Data transformation then categorized more than 3100 citations on frequency analyses, globally and by domain, visit, and participant. Regarding quantitative results, there was no clear evidence that any of the three PRO tools captured positive changes as a result of NAC treatment.

Conclusion Qualitative results showed a comprehensive characterization of HRQoL in this population based on a symptom/ patient-centered approach. These findings will inform future studies. Furthermore, given the similar findings across our multiple methods and endpoints, the introduction of MMR may be a valuable, complementary approach to clinical trials. MMR may be especially useful to incorporate in order to address and follow the FDA's guidance and prioritization on the inclusion of affected individuals' perspectives in clinical trials.
\end{abstract}

Keywords Neuromuscular disorders $\cdot$ Natural history study $\cdot$ Clinical trial $\cdot$ Health-Related Quality of Life $\cdot$ Mixed methods

Electronic supplementary material The online version of this article (https://doi.org/10.1007/s11136-020-02428-2) contains supplementary material, which is available to authorized users.

Carlos Capella-Peris

carlos.capellaperis@mail.nih.gov; capellac@uji.es

1 Neuromuscular Symptoms Unit, Tissue Injury Branch, National Institute of Nursing Research, National Institutes of Health, Bethesda, MD, USA

2 Biostatistics and Clinical Epidemiology Service, National Institutes of Health Clinical Center, Bethesda, MD, USA

\section{Introduction}

In the era of precision medicine, the importance of patientcentered data is becoming increasingly recognized by regulators, clinicians, and the research community. Since the Food and Drug Administration's (FDA's) Guidance for Industry on Patient-Reported Outcome (PRO) Measures [1] and Patient-Focused Drug Development [2,3], there has been a shift from traditional methods solely focused on clinical study goals to the incorporation of participant experience outcomes. Research methods are now including the patient perspective early in order to ensure (and validate) that any changes measured are meaningful to those patients [4]. PROs can provide important insights into disease symptoms 
or treatment side effects (e.g., pain, fatigue, or anxiety); functional outcomes (e.g., physical, social, emotional, etc.); and/or multidimensional assessment of the impact of both disease and treatment on physical, psychological, and social well-being, also known as Quality of Life (QoL) [5].

PROs can be used to generate both quantitative (e.g., questionnaires) and qualitative (e.g., interview, journals) data. Mixed methods research (MMR) employs quantitative and qualitative approaches to provide a more comprehensive understanding of findings. MMR has been proposed as a best practice to inform Patient-Centered Outcomes Measures in rare diseases where quantitative methods alone have found challenges [6]. Thus, MMR was employed during a combined natural history study and clinical trial of $N$-acetylcysteine (NAC) in Ryanodine 1-Related Myopathies (RYRI-RM) to facilitate an understanding of participants' characterization of their condition and to identify any subjective improvements post-intervention. RYRI-RM comprise the most common of the non-dystrophic neuromuscular disorders with a pediatric incidence of $>1: 90,000$ in the United States, yet there are no FDA-approved treatments [7, 8].

\section{Materials and methods}

\section{Standard protocol approvals, registrations, and patient consents}

The RYR1 NAC clinical trial consisted of two components; a prospective natural history assessment and a parallelgroup, randomized, double-blind, placebo-controlled trial. The study was conducted at the National Institutes of Health (NIH) Clinical Center, Bethesda, MD, USA between 2015 and 2017. The clinical trial is registered in the U.S. National Library of Medicine (NCT02362425). All procedures were approved by the NIH Combined Neuroscience Institutional Review Board (CNS IRB), and an Independent Monitoring Committee was established to oversee trial safety. All participants and parents of participants $<18$ years of age provided written informed consent, according to the Declaration of Helsinki, before enrollment. Assent was also obtained for those $<18$ years of age. Participants attended three study visits at the NIH: baseline (0 months), pre-intervention (6 months), and post-intervention (12 months). Participants also completed a follow-up phone call (18 months). Randomization was performed at the end of the second visit. For more information regarding the clinical trial, see the following publication [9].

\section{Study design and procedures}

Common RYRI-RM clinical manifestations include delayed motor milestones, hypotonia, scoliosis, ophthalmoplegia, respiratory insufficiency, myalgia and rhabdomyolysis, exertional heat stroke/myalgia, proximal/axial muscle weakness, and fatigue $[10,11]$. Affected individuals reported fatigue as one of the more pervasive symptoms [12]. NAC was shown to improve measures of physical endurance and force production in a preclinical setting $[13,14]$. Therefore, we performed a comparison of quantitative and qualitative PRO data to identify subjective improvements from NAC treatment (PharmaNAC®, BioAdvantex Pharma Inc., Toronto, Ontario, Canada). We used the National Institute of Nursing Research (NINR) Symptom Science Model [15] to guide the investigation of how patient-reported symptoms affect Health-Related Quality of Life (HRQoL) in RYRI-RM.

Our mixed method design uses methodological triangulation. In this design the qualitative and quantitative components are typically undertaken simultaneously and weighted equally, seeking convergence of findings to enhance the interpretability of a single phenomenon [16, 17]. Methodological triangulation is used to provide a complementary view from qualitative and quantitative approaches, balancing the strengths and weaknesses of both.

Qualitative data were collected via semi-structured interviews at each visit to characterize natural history of HRQoL in ambulant individuals with $R Y R I-\mathrm{RM}$ prior to randomization and to analyze drug effects post-intervention. Thus, individual and collective viewpoints were addressed. The use of this design has been previously implemented in HRQoL and Neuromuscular Disorders (NMD) [18-20] research. One hundred-seven semi-structured interviews were conducted using open-ended questions (Table 1) (i.e., forty-six from baseline visit, thirty-four from pre-intervention, and twenty-seven from post-intervention). Semi-structured interviews were recorded and transcribed verbatim to conduct thematic analysis $[19,20]$ through a multiphase approach, open-coding and axial-coding [21]. As RYRI-RM are slowly progressive diseases, this was considered when analyzing HRQoL and treatment effects. Three researchers conducted qualitative analysis. All researchers codified an initial set of five semi-structured interviews to identify common areas of interest (domains/subdomains) and to assess agreement. Once agreement was confirmed, the remaining interviews were distributed among the researchers for analysis. A balanced number of interviews were provided to each researcher considering the following variables: males/females, adults/ children, and pre-intervention/post-intervention. Three additional meetings were conducted to discuss the inclusion of new domains/subdomains until saturation was achieved [22]. Differences in coding were resolved through consensus and tighter definitions of subdomains [23]. Likewise, interpretation and selection of quotes for publication was also discussed to ensure agreement among researchers.

We transformed qualitative data into quantitative results, a standard procedure in mixed methods research, counting 
Table 1 Open-ended questions from semi-structured interviews

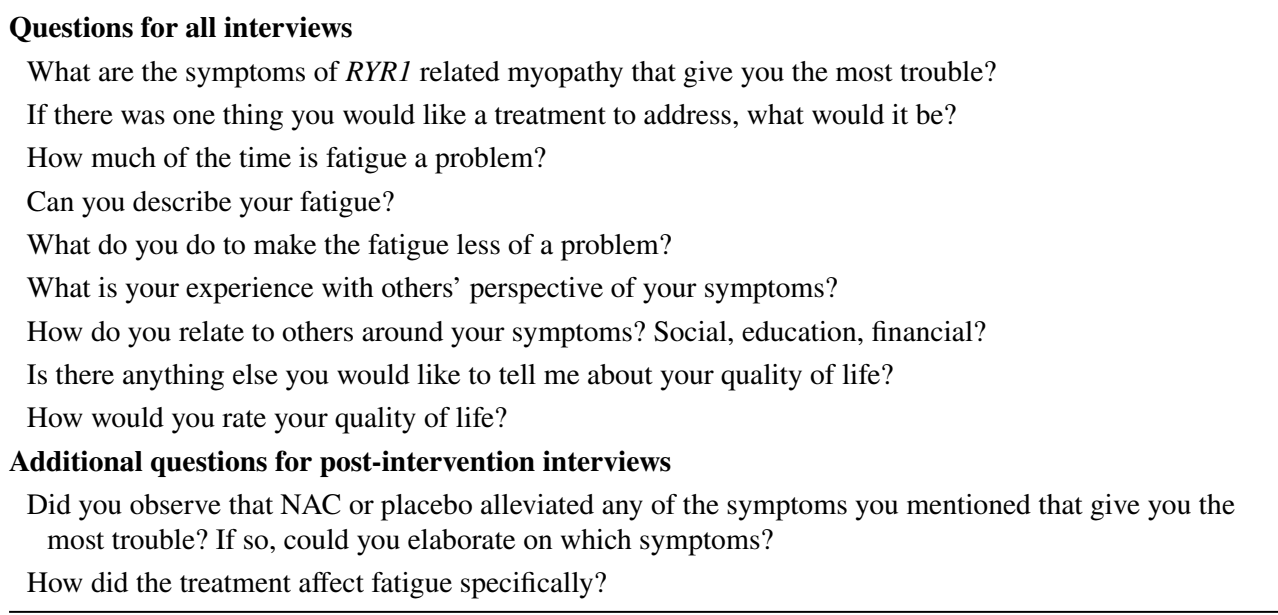

the number of times with which each domain/subdomain was mentioned in semi-structured interviews [19, 24, 25]. The average and percentage of citations was also calculated for each domain/subdomain. This procedure was performed globally (i.e., considering all data), by domain (i.e., analyzing every identified domain separately), by visit (i.e., considering the records for each visit independently), and by participant (i.e., evaluating data provided for each participant individually). When necessary, the percentage score was normalized to compare domains/subdomains. Additionally, a word frequency analysis was also completed. Results from that analysis were presented through a word cloud image [26]. Qualitative data were also used to evaluate treatment effects via subjective self-assessment. Because we were evaluating treatment-related improvements in this analysis, a binary score was assigned to participants based on their comments on post-intervention interviews (i.e., "positive effect" $[+1]$ vs "no change" [0]). Disease progression was assessed during qualitative analysis, where only minor side effects were reported. Subjective self-assessment was provided by participants under 18 years of age and/or their parents, when necessary. Counts of positive and no change reports were compared between the two treatment groups (NAC vs. placebo) via Fisher's exact test.

Finally, the Patient-Reported Outcomes Measurement Information System (PROMIS) [27] and Quality of Life in Neurological Disorders (Neuro-QoL) [28] scores were gathered. Twenty domains from PROMIS and Neuro-QoL were analyzed separately via t-test (i.e., anger, anxiety, depression, fatigue, mobility, pain, peer relations, social, and upper extremity for PROMIS; and anger, anxiety, applied cognition, depression, fatigue, mobility, pain, participate in SRA, social relations, stigma, and upper extremity for Neuro-QoL, respectively). For PROMIS and Neuro-QoL, the change in $T$-score between pre- and post-treatment was analyzed. $T$-score distributions rescale raw scores into standardized scores with a mean of 50 and a standard deviation (SD) of
10. More specifically, the change in $T$-score between Month 12 and Month 6 was calculated (Month 12 minus Month $6)$. Month 0 was used instead of Month 6 whenever Month 6 was missing. In some cases, the participant had both a self-report and a parent proxy PROMIS or Neuro-QoL $T$-score on the same domain. Also, Case 33 had two different PROMIS or Neuro-QoL $T$-scores on the same domain. In these cases, and within each specific domain, the average change was calculated and analyzed. To compare the treatment groups (NAC and placebo), a $t$-test on the $T$-score change was performed.

SAS version 9.4 software (SAS Institute Inc., Cary, NC) was used to conduct all statistical analysis and Nvivo version 12.3 software was used to perform qualitative and data transformation analyses.

\section{Study goals}

The primary goal of this study was to characterize HRQoL in ambulant individuals with $R Y R 1-\mathrm{RM}$ to support future research. Questionnaire data (i.e., $T$-scores generated by PROMIS and Neuro-QoL instruments) have shown limitations assessing rare diseases [6]. As a secondary goal, this study thus aimed to determine if open-ended data (gathered via subjective self-assessment interviews) captured additional information to detect changes to HRQoL in ambulant individuals with $R Y R 1$-RM post-NAC treatment.

\section{Research questions and hypothesis}

$Q_{1} \quad$ What are the major concerns/issues regarding HRQoL in ambulant individuals affected with $R Y R 1-\mathrm{RM}$ ?

$Q_{2} \quad$ How do ambulant individuals with $R Y R$ l-RM describe the major concerns/issues affecting their HRQoL? 
Our research hypothesis was that subjective self-assessment will capture additional information and complement PROMIS and Neuro-QoL instruments to detect changes in HRQoL in ambulant individuals with RYRl-RM when comparing the NAC and placebo groups.

\section{Participants}

One hundred-fifty individuals were screened for participation in this study, of which 53 were eligible and enrolled (Fig. 1). Of these, 47 participants completed at least one interview in which we were able to perform qualitative analysis. Only participants with complete data from all three tools (subjective self-assessment, PROMIS, and Neuro-QoL) were included in quantitative analysis to avoid potential confounding factors of varying data from different participants. Therefore, quantitative analysis included 26 participants -13 in each of NAC and placebo groups.
Demographic information of participants is presented in Table 2.

\section{Results}

\section{Qualitative analysis}

Five domains and 33 subdomains were identified as areas of interest in participants with $R Y R 1-\mathrm{RM}$ regarding their HRQoL. Quality of life domain comprised 11 subdomains (i.e., anxiety, career/financial impact, diagnostic journey, disease progression, frustration, general assessment, positive aspects, sadness, self-care/home maintenance, selfesteem, and social impacts); adaptive strategy contained six subdomains (i.e., accommodation, equipment, help from others, physical behaviors, physical therapy, and psychological strategy); symptoms covered 12 subdomains (i.e.,

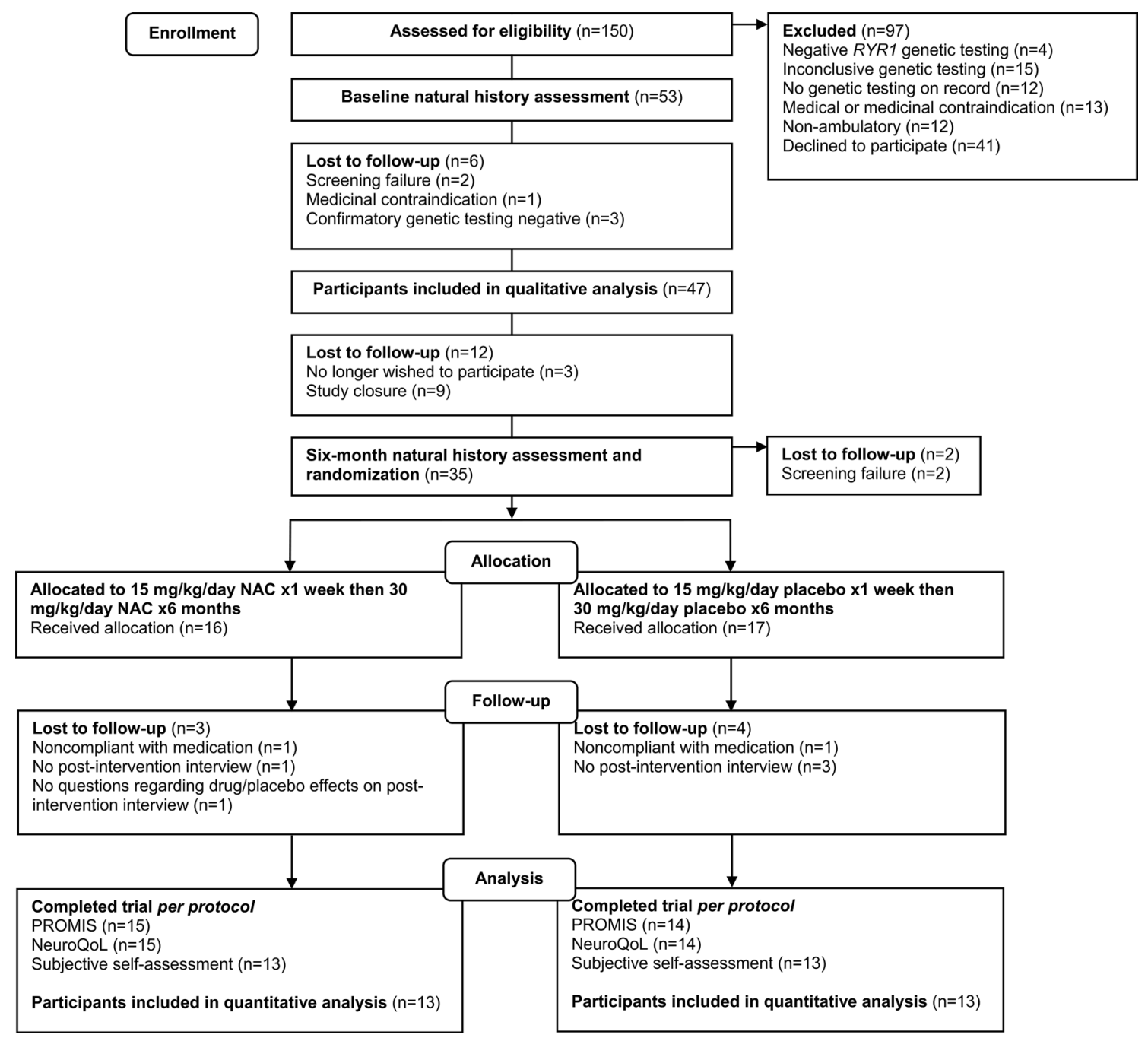

Fig. 1 Consort diagram of study flow 
Table 2 Participant demographic information

\begin{tabular}{|c|c|c|c|c|c|}
\hline Variables & Overall participants & Qualitative analysis & Quantitative analysis & NAC group & Placebo group \\
\hline$N$ & 53 & 47 & 26 & 13 & 13 \\
\hline Cohort $\%$ & $100 \%$ & $89 \%$ & $50 \%$ & $25 \%$ & $25 \%$ \\
\hline \multicolumn{6}{|l|}{ Gender } \\
\hline Female & $29(55 \%)$ & $27(57 \%)$ & $15(58 \%)$ & $8(62 \%)$ & $7(54 \%)$ \\
\hline Male & $24(45 \%)$ & $20(43 \%)$ & $11(42 \%)$ & $5(38 \%)$ & $6(46 \%)$ \\
\hline \multicolumn{6}{|l|}{ Age } \\
\hline Total & $29.8 \pm 17.6$ & $29.6 \pm 17.1$ & $28.3 \pm 17.2$ & $32.8 \pm 16.3$ & $23.8 \pm 17.6$ \\
\hline Adults $(>18)$ & $\begin{array}{l}35(66 \%) \\
39.9 \pm 12.7\end{array}$ & $\begin{array}{l}31(66 \%) \\
39.4 \pm 12.4\end{array}$ & $\begin{array}{l}15(58 \%) \\
41.3 \pm 9.8\end{array}$ & $\begin{array}{l}9(69 \%) \\
42.4 \pm 7.3\end{array}$ & $\begin{array}{l}6(46 \%) \\
39.7 \pm 13.2\end{array}$ \\
\hline Peds $(<18)$ & $\begin{array}{l}18(34 \%) \\
10.2 \pm 2.7\end{array}$ & $\begin{array}{l}16(34 \%) \\
10.5 \pm 2.8\end{array}$ & $\begin{array}{l}11(42 \%) \\
10.5 \pm 2.3\end{array}$ & $\begin{array}{l}4(31 \%) \\
11.0 \pm 2.9\end{array}$ & $\begin{array}{l}7(54 \%) \\
10.1 \pm 2.0\end{array}$ \\
\hline \multicolumn{6}{|l|}{ Ethnicity } \\
\hline White & $47(89 \%)$ & $42(89 \%)$ & $25(96 \%)$ & $12(92 \%)$ & $13(100 \%)$ \\
\hline Black & $6(11 \%)$ & $5(11 \%)$ & $1(4 \%)$ & $1(8 \%)$ & $0(0 \%)$ \\
\hline \multicolumn{6}{|l|}{ Location } \\
\hline Northeast & $10(19 \%)$ & $10(21 \%)$ & $4(15 \%)$ & $1(8 \%)$ & $3(23 \%)$ \\
\hline Southeast & $9(17 \%)$ & $9(19 \%)$ & $7(27 \%)$ & $5(39 \%)$ & $2(15 \%)$ \\
\hline Midwest & $10(19 \%)$ & $5(11 \%)$ & $2(8 \%)$ & $2(15 \%)$ & $0(0 \%)$ \\
\hline Southwest & $4(7 \%)$ & $4(9 \%)$ & $3(12 \%)$ & $2(15 \%)$ & $1(8 \%)$ \\
\hline West & $10(19 \%)$ & $10(21 \%)$ & $4(15 \%)$ & $2(15 \%)$ & $2(15 \%)$ \\
\hline Abroad & $10(19 \%)$ & $9(19 \%)$ & $6(23 \%)$ & $1(8 \%)$ & $5(39 \%)$ \\
\hline
\end{tabular}

compounding illness-conditions, fatigue, fine motor, heat intolerance, mobility, muscle spasms, numbness, pain, respiratory difficulties, rhabdomyolysis, scoliosis, and weakness); future thoughts comprised two subdomains (i.e., fears and treatment desires); and post-intervention included two subdomains (i.e., treatment benefits and side effects). The most representative domains/subdomains, by their importance and depth are provided in Table 3 to reveal participants' experiences/opinions. In this table, researchers' interpretations of participants' interviews are labeled with domain and subdomain. Transcripts of participants' comments are also included. A reference code noting deidentified case number, visit, researcher initials, and Nvivo transcript reference number follows each quote.

\section{Data transformation}

More than 3100 citations were categorized. The results of the global analysis and analysis by domain are displayed in Fig. 2. Examples of results for analyses by visit and participant are available as Supplemental Data (Tables e-1 to e-3 and Fig. e-1).

Results from the word frequency analysis were summarized as a word cloud (Fig. 3).

Post-intervention comments transformed into a binary value (positive effect or no change) revealed a higher frequency of subjective positive changes observed in the NAC group versus the placebo group (69\% versus 38\%) and a higher frequency of subjective no change observed in the placebo group versus the NAC group (62\% versus $31 \%)$, but the $p$ value from Fisher's exact test (two-sided) was 0.24.

\section{Quantitative analysis}

Only three domains from the Neuro-QoL instrument, anxiety, stigma, and fatigue, showed some evidence of improvement in $T$-scores in the NAC group compared to placebo. The $p$ values for these domains ranged between 0.014 and 0.034 , whereas all other $p$ values for the Neuro-QoL and PROMIS were above 0.05 (Table 4). These $p$ values were not corrected for multiple analyses.

\section{Discussion}

Based on our qualitative results, we were able to identify and characterize major HRQoL concerns/issues in ambulant individuals affected with RYRI-RM. The Quality of life domain was the most relevant for participants when discussing their HRQoL. The most emphasized subdomain was social impacts, depicting specific perceptions, limitations, and deficits of understanding due to the disease. For example, mildly-affected participants were widely accused of being "lazy" or "liars", which is consistent with previous 
Table 3 Transcripts from the most representative domains/subdomains

Quality of life > Social impacts: the most remarkable issue reported in interviews was a lack of other people's understanding of $R Y R 1$ myopathies. Our first thought was to attribute this to the fact that $R Y R 1-\mathrm{RM}$ is rare and thus not understood by the general population. However, the interviewees' comments focused primarily on their lack of apparent symptoms. As other people are not able to easily recognize a myopathy at first glance in milder cases, they tend to minimize the physical effects of the disease. This observation highlighted the conundrum of displaying visual symptoms. For this reason, participants struggled to explain their condition, typically choosing one of these options: giving very little information or saying nothing at all, using the name of a similar disease that is widely known such as Amyotrophic Lateral Sclerosis (ALS), or stating they have a "muscle condition" and describing its main symptoms (e.g., fatigue, pain, weakness, etc.). In addition, participants indicated similar situations happened on a daily basis, increasing the effect of this social issue. This subdomain revealed direct connections with several other domains such as symptoms, adaptive strategy, and future thoughts.

“when I'm standing up, generally I think it doesn't, people don't perceive that there's a challenge that I have. When I have things like, I had it with the shuttle from the airport where I had to ask the gentleman to put down the wheelchair lift, and he kinda looked at me like 'Well, you don't look like anything's wrong', that sort of thing. Having to explain that I have a form of, I have muscular dystrophy, and I simply can't do stairs or it's too difficult for me to do, is generally how I explain it. Whether or not it's believable... I think some folks don't quite understand it. If something doesn't look wrong, it can't be wrong" <C04VA_MMC $>$ Ref. [1]

Adaptive strategy > Physical behaviors: the most common solution mentioned for the main symptoms (e.g., fatigue, weakness, pain) was simply to cease activity and rest. This solution not only displayed a motor limitation, but also required increased organizational skills, as affected individuals needed more time to complete activities. Participants had to consider which activities were to take precedence, because they were not able to partake in all physical activities in which they would have liked to participate. Although these situations varied according to level of exertion, they reported a continuous and high degree of fatigue. This showed clear links between fatigue, psychological strategy, career/financial impact, and social impact subdomains.

“when I can't go anymore, I just stop, basically. And then I usually don't try to do any more that day, and just work on it another day a little bit... It will help some, but if I like, get to feeling a little better and then I start back, it will get worse. But it kind of depends on to what level of fatigue I get" (NAC) < C17VC_MMC > Ref. [1]

Adaptive strategy > Psychological strategy: whereas the previous paragraph explained how individuals with RYR1-RM faced effects of the disease from a functional approach, participants also used a psychological approach. The most common strategies included increased organization, learning from previous experiences, and applying problem solving to each situation. These strategies required a constant focus on how to improve their QoL, which actually reduced QoL. This created additional psychological fatigue that compounded the physical fatigue they experienced. There was also a tendency to compare themselves with healthy individuals, bringing attention to the problems they faced and feelings of frustration with limitations. This subdomain had connections with psychological factors, such as fears, anxiety, frustration, and self-esteem, as well as physical aspects, such as fatigue, weakness, pain, or physical behaviors.

"It makes any situation that much more complicated, 'cause I have to take into account will I be able to do it? Will there be an obstacle? I think I plan ahead a lot more. I think like almost a military strategist in terms of what will happen in this event? Will there be stairs? Will I have to sit on the floor? If I have to do that, how will I handle that? It's a lot more of that. It's just draining in a way to constantly have to, as opposed I think people who don't aren't affected by things like this and can flow through life without having to worry about those things" $<\mathrm{C} 36 \mathrm{VA}$ ICC $>$ Ref. [5]

Symptoms > Fatigue: participants reported their symptoms affected them on a daily basis, revealing the frequency of the disease's impact. Indeed, most said these symptoms, not only fatigue but also weakness and pain, had a continuous influence in their lives, revealing the difficulties associated with their physical condition. For this reason, they limited and organized their daily activities, drastically reducing their ability to perform motor tasks. In addition, participants described their fatigue, using words such as "depletion", "exhausted", "empty", "drained", "having zero left in the tank", which points to the benefit of potentially improving fatigue. As in previous cases, the concept of fatigue spread to other subdomains such as physical behavior, self-care/home maintenance, career/financial impact, and mobility.

"Probably daily. I don't really wake up feeling rested usually, and then I can usually do my daily morning stuff. I go to work. All that stuff, and then in the afternoon, typically, kind of when I'm winding down in the evenings. Sometimes, I'll need to take a nap before I do things around the house for kind of, like, round two at the house... It's just kind of like a general overall sleepiness. So, it's not like, I don't experience particular muscle fatigue in, like, a muscle group. It's just, if I do everything I need to do for the day, I'll come home, and I'll be tired so, I'll need to rest. It's not necessarily a muscular tired. It's more all over general fatigue" < C09VA_MMC > Ref. [3]

Future thoughts $>$ Treatment desires: in general, participants expressed the symptoms they wanted most to be addressed by a potential treatment were fatigue and weakness. Having impairments in these areas limited physical performance considerably. Despite this, participants focused more on what they could do or what they could improve instead of dwelling on limitations. Similarly, they were committed to helping others rather than solely considering their own benefit. This revealed both a positive approach and a sense of social belonging related to the disease. All this information connected to several domains/subdomains in our research: quality of life, fatigue, social impact, positive aspects, and disease progression, strengthening the importance of the identified areas of analysis.

"I don't know if this would be the same thing or not, but I would say energy or zero fatigue... I am hopeful about this trial. And, while I don't know that it will provide a huge quality of life improvement for me, down the road if this and other trials help my children to have a much easier life, and I think that's why Brandon is participating... That I think those are all good things. And so, recently I went with my son to, he has a different neurologist than I do, and he was very hopeful about if not treatments, at least ways that we can manage muscular dystrophy going forward. It's not quite the hopeless condition that it once was" $<\mathrm{C} 19 \mathrm{VB} \_\mathrm{CCP}>\mathrm{Ref} .[1-2]$ 
Table 3 (continued)

Post-intervention > Treatment benefits: several participants reported positive results of using NAC for treatment in RYR1-RM. The most valuable benefits were related to reducing fatigue and increasing strength. Additionally, several patients also experienced reduced pain, which could be indirectly produced by a reduction of tiredness and/or an increase of strength. These observations revealed some gain in their motor performance that in turn implied improvement in independence because they required less support in physical activities. Thus, some participants expressed access to a new and larger range of activities as well as a greater availability of time, suggesting that NAC impact was not limited to the physical domain. As expected, the strongest relationship for this subdomain was linked to the domain of symptoms, however, additional connections with other subdomains, such as physical behaviors, general assessment, or disease progression, were also present.

“It lessened it, I think. I don't think I'm near as tired... I still can't go up a flight of stairs without a handrail. But maybe it's not quite as hard... I think there's been little things that at the end of the day have equaled up to me not being as tired and as fatigued as I was because the completely washed out, complete drained, just can't go no more" (NAC) $<\mathrm{C} 32 \mathrm{VC} \_\mathrm{CCP}>\mathrm{Ref}$. [1]

"the weakness seems to be somewhat better. But it still exists, but it seems noticeably... I noticed it more in the first couple months, and then it seemed like it kind of hit a plateau and stayed at the same level throughout until the end... Like, yesterday, and since I've been on the medicine, I do notice that my recovery has been quicker than normal" (NAC) $<$ C17VC_MMC $>$ Ref. [1-3]

"They ruled my day from the moment I woke up in the morning. I woke up in pain every single morning. It was really significant. I wasn't aware of how severe my pain was even though I had - even knowing I was in pain, I couldn't really assess how severe it was until it was gone, because I had it my whole life. I was born with that level pain. I never had relief until six months ago. And it was breathtaking how dramatic it was not to have muscle pain, especially not to have ankle pain, to be able to wake up and then get up out of bed immediately instead of waking up and waiting up to two hours before I could get out of bed, because of the pain... And now I just, I go through the whole day. I don't even really have to think about it... But then to have to lie there staring at the ceiling and thinking about things and imagining what I would do and having to deal emotionally that frustration of wanting to get up and live and take care of my children and work" $(\mathrm{NAC})<\mathrm{C} 38 \mathrm{VC} \_\mathrm{CCP}>\mathrm{Ref}$. [1]

Post-intervention > Side effects: participants reported very few side effects regarding RYR1-RM treatment with NAC. Side effects that were reported were minor (e.g., acid reflux or headaches). In one case, a participant reported potential increased fatigue, but later contradicted that report. This supported the minimal risk involved on this research. Due to the specificity of this subdomain there were no links to other areas of the study.

"I had extreme fatigue and I don't know if it's just because it was the weather change or if it was the drug or what, and then I've had a shift where I've had less fatigue... I do see some improvements. Like, I was able to go up the stairs yesterday for the PT trial without holding on the railing, which I have never been able to do" (NAC) $<\mathrm{C} 01 \mathrm{VC} \_\mathrm{CCP}>\mathrm{Ref}$. [1]

"From the medicine I had? Headaches" (placebo) $<$ C24VC_ICC $>$ Ref. [1]

"Some acid reflux" (placebo) $<$ C34VC_MMC $>$ Ref. [1]

For post-intervention interviews, drug assignment precedes the reference code. Pseudonyms were used to protect patient identity

reports in qualitative research on NMD [29, 30]. This finding highlights the importance of establishing positive interactions to enhance HRQoL. General assessment and positive aspects also stood out as notable subdomains. Participants were very optimistic in their appraisals of their QoL as a result of internal acceptance, focus on improvement, encouraging experiences, and support of family and friends. This depicts a high level of resilience in facing their condition, which is a well-documented response to adversity in physical and mental health $[31,32]$. The remaining subdomains, however, received moderate-to-little attention, highlighting that, globally, they were less important to participants than the previous subdomains. For example, negative experiences were linked to specific economic-work situations, unhappy moments, the progression of the disorder, daily care and domestic activities, annoyance reactions, difficulties in being diagnosed, and uneasy feelings. Most of these have been described in RYRI-RM [12, 33, 34] and other NMD [35, 36]. However, it is surprising that self-esteem was not explored in depth in participant interviews. This indicates very little effect on self-esteem, which is in contrast to previous research in individuals with NMD [37, 38]. It is also worth noting that contrary results regarding anxiety have been reported in the RYRI-RM population [12]. All subdomains for quality of life revealed connections with the other identified areas of interest, especially with adaptive strategy and symptoms. This observation is based on the close interaction between QoL and HRQoL concepts [39].

The second domain was adaptive strategy, which gave insight into several coping strategies employed by participants. Most of these fell into the subdomains of physical behaviors and psychological strategy, which described the approaches taken to address motor restrictions (e.g., limiting physical activities, increasing rest periods, using external support, etc.) and psychological implications (e.g., planning ahead, scheduling energy, remaining positive, etc.). Implications in both areas were also reported recently for individuals with RYR 1-RM [12] and HRQoL research in NMD [24]. Accommodation, equipment and help from others attained similar emphasis, disclosing the need for additional assistance from institutions, tools, and people, respectively. These factors revealed a lack of independence for participants with RYR1-RM, which is a key aspect for the NMD population in general [20]. Surprisingly, physical therapy was scarcely mentioned by participants, with minor mentions of therapeutic and occupational interventions like horseback riding. 


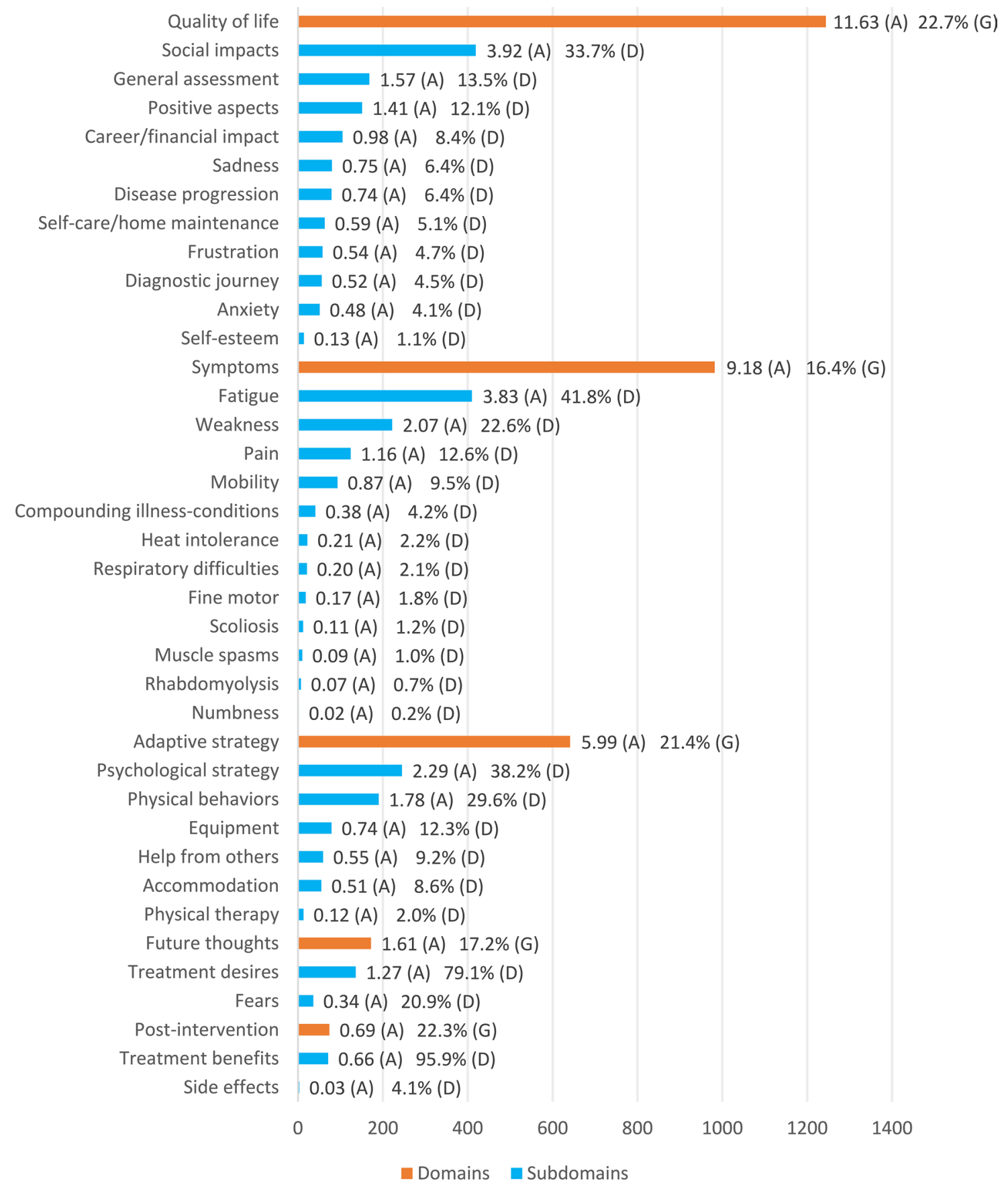

Fig. 2 Data transformation results for global and domain analyses $(N=107)$. Total count (whole bar), average (A), global percentage for each domain $(\mathrm{G})$, and domain percentage for each subdomain (D) are

We posit this is due to the relative normality of this type of therapy in this population. However, this could be also due to differences in access to physical therapy services. This issue should be explored in depth in future research. Every adaptive strategy was linked to the specific symptoms of each participant, indicating the strong relationship between both domains, as might be expected. displayed. The percentages were normalized for the number of subdomains in each domain and given that post-intervention comments were only recorded on post-intervention interviews

Another important domain was symptoms. The most influential subdomain was fatigue, followed by weakness. This is consistent with documented clinical effects of RYRI-RM [8, 11, 12, 33, 40-44]. Pain and impaired mobility were secondarily important in this study and were mainly described as consequences of fatigue and weakness. Likewise, there are several antecedents of pain 


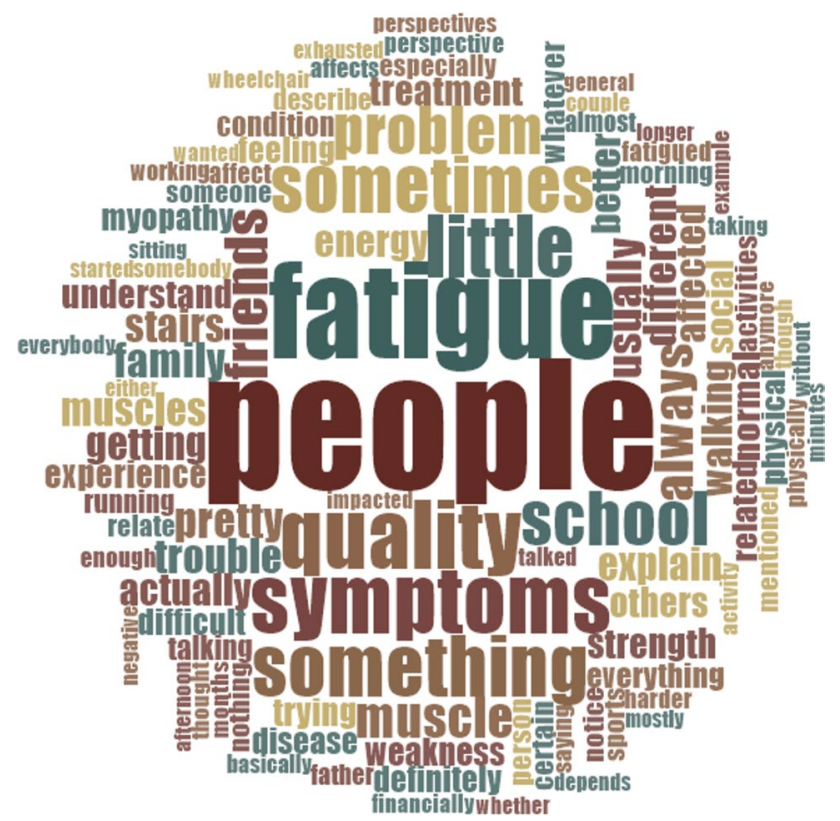

Fig. 3 Word cloud summarizing word frequency analysis. Larger font sizes indicate a higher frequency of mentions; smaller font sizes a lower frequency

and impaired mobility in affected individuals with RYRIRM $[8,10,12,34,43]$. The remaining subdomains were less widely reported and included issues more specific to individuals such as additional complications, malignant hyperthermia experiences, respiratory issues, hand dexterity problems, and abnormal curvatures of the spine, aligning with manifestations of RYRI-RM [8, 11, 12, 33, 40, 42, 44-46]. Rhabdomyolysis was not described in depth. However, it is possible that participants reported rhabdomyolysis involvement within the weakness subdomain, as previous research notes the importance of this symptom for individuals with RYRl-RM [10, 45, 46]. Finally, muscle spasms and numbness were touched on but not elaborated, suggesting they are not paramount. Despite this, these symptoms have been previously reported [43, 46]. Every aspect of the symptoms domain displayed a high influence on other subdomains, reinforcing the whole construct and emphasizing the use of the Symptom Science Model [15].

The domain future thoughts captured feelings about events to come. The Fears subdomain contained concerns about potential issues, including limitations on activities, worsening symptoms, or genetic implications for children. Treatment desires, a subdomain consistent with recent qualitative data in NMD [35], was essentially focused on improving HRQoL as a result of increasing physical performance. These findings could be interpreted as a dualistic display (i.e., positive vs negative) of treatment expectations [47]. This domain was closely related to other identified domains/ subdomains such as symptoms and disease progression, bolstering their identification as areas of interest.

Finally, the post-intervention domain captured individual impressions of treatment effect after the NAC/placebo intervention, addressing our research hypothesis regarding whether or not these data captured additional information complementing PROMIS and Neuro-QoL instruments to detect changes in HRQoL in ambulant individuals with RYRI-RM post-treatment. These reactions were categorized as positive or no change. Participants' feedback revealed valuable treatment benefits, most of which were related to increased levels of energy and strength, which are consistent with preclinical effects of NAC on ryrl mutant zebrafish and mice $[13,14]$. However, results from our quantitative analysis of Neuro-QoL and PROMIS $T$-scores were inconclusive. Social and psychological profits were also reported [39]. Only minor side effects were noted, providing further evidence that NAC is safe and well tolerated in this population. As in previous subdomains, both options were directly connected to the specific symptoms of each participant, which indicates a straightforward relationship between both domains.

Regarding data transformation, analysis by domain exposed a clear imbalance in future thoughts and postintervention domains, recording more quotes for treatment desires and treatment benefits subdomains. This highlighted that participants with RYRI-RM tended to show more positive attitudes towards their HRQoL in comparison to their family, caregivers, or external observers, which is known as the "disability paradox" [48]. The "disability paradox" has been noted in previous research on NMD [49, 50]. Quality of life, adaptive strategy, and symptoms domains were relatively balanced. However, social impacts, psychological strategy, and fatigue subdomains scored higher number of comments for each case. These findings are consistent with the qualitative data, reinforcing the results obtained via both approaches.

The word cloud revealed the presence of three main groups of terms "people, family, friends, school, person, others"; "fatigue, energy, weakness, myopathy, affected, symptoms"; and "quality, little, problem, better, harder, difficult, without, describe". Those words reemphasize the importance of several domains/subdomains such as social impacts; symptoms and physical behaviors; and quality of life and adaptive strategy, respectively. These results also coincide with our previous findings from qualitative analysis.

Global analysis, involving transformed data, found balanced percentages of all identified domains, considering the number of subdomains in each case and that postintervention comments were only reported on post-intervention interviews. This outcome differed slightly from qualitative data, where quality of life, adaptive strategy and symptoms were more relevant, and suggested a similar 
Table 4 Results from $t$-test for PROMIS and Neuro-QoL instruments (in order of lowest to highest $p$ value)

\begin{tabular}{|c|c|c|c|c|c|c|c|c|}
\hline Instrument-domain & $\begin{array}{l}N \text { in } \\
\text { NAC } \\
\text { group }\end{array}$ & $\begin{array}{l}N \text { in } \\
\text { Placebo } \\
\text { group }\end{array}$ & $\begin{array}{l}\text { Average } \\
\text { change } e^{\mathrm{a}} \text { in NAC } \\
\text { group }\end{array}$ & $\begin{array}{l}\text { Average change } \\
\text { in Placebo group }\end{array}$ & $\begin{array}{l}\text { Difference } \\
\text { between } 2 \\
\text { groups }\end{array}$ & $\begin{array}{l}\text { Lower limit } \\
\text { of difference }\end{array}$ & $\begin{array}{l}\text { Upper limit } \\
\text { of difference }\end{array}$ & $\begin{array}{l}p \text { value }^{\mathrm{b}} \\
\text { for zero } \\
\text { difference }\end{array}$ \\
\hline Neuro-QoL-anxiety & 12 & 12 & -5.82 & -0.11 & -5.71 & -10.16 & -1.26 & 0.014 \\
\hline Neuro-QoL-stigma & 12 & 12 & -5.15 & 0.03 & -5.17 & -9.81 & -0.53 & 0.030 \\
\hline Neuro-QoL-fatigue & 12 & 12 & -6.16 & 0.14 & -6.30 & -12.09 & -0.51 & 0.034 \\
\hline PROMIS-peer relations & 3 & 5 & -4.75 & 3.81 & -8.57 & -19.39 & 2.26 & 0.101 \\
\hline PROMIS-pain & 12 & 11 & -7.54 & -1.65 & -5.90 & -13.50 & 1.70 & 0.116 \\
\hline $\begin{array}{l}\text { Neuro-QoL-participate } \\
\text { in SRA }\end{array}$ & 9 & 6 & 3.43 & 0.28 & 3.15 & -2.04 & 8.34 & 0.148 \\
\hline Neuro-QoL-mobility & 9 & 6 & 2.85 & -0.37 & 3.21 & -1.36 & 7.78 & 0.153 \\
\hline PROMIS-fatigue & 12 & 11 & -7.79 & -2.58 & -5.21 & -12.94 & 2.51 & 0.168 \\
\hline PROMIS-mobility & 12 & 11 & 1.92 & -0.80 & 2.72 & -1.31 & 6.74 & 0.175 \\
\hline PROMIS-social & 9 & 6 & 6.35 & 1.32 & 5.03 & -4.19 & 14.25 & 0.185 \\
\hline $\begin{array}{l}\text { Neuro-QoL-upper } \\
\text { extremity }\end{array}$ & 9 & 6 & 2.29 & -0.53 & 2.82 & -2.57 & 8.21 & 0.200 \\
\hline PROMIS-upper extremity & 12 & 11 & 2.86 & 1.10 & 1.77 & -1.97 & 5.50 & 0.326 \\
\hline $\begin{array}{l}\text { Neuro-QoL-social rela- } \\
\text { tions }\end{array}$ & 3 & 2 & 6.76 & 1.81 & 4.95 & -11.48 & 21.38 & 0.408 \\
\hline $\begin{array}{l}\text { Neuro-QoL-applied } \\
\text { cognition }\end{array}$ & 3 & 2 & 3.76 & -2.96 & 6.72 & -16.17 & 29.62 & 0.419 \\
\hline Neuro-QoL-Pain & 3 & 6 & -3.50 & -0.79 & -2.72 & -12.48 & 7.05 & 0.532 \\
\hline Neuro-QoL-depression & 12 & 8 & -2.62 & -0.63 & -1.99 & -8.57 & 4.60 & 0.534 \\
\hline PROMIS-anxiety & 12 & 12 & -3.93 & -2.79 & -1.14 & -6.11 & 3.83 & 0.639 \\
\hline Neuro-QoL-anger & 3 & 6 & -4.74 & -3.30 & -1.44 & -14.76 & 11.87 & 0.805 \\
\hline PROMIS-anger & 12 & 11 & -3.62 & -3.12 & -0.50 & -6.93 & 5.94 & 0.874 \\
\hline PROMIS-depression & 12 & 12 & -3.66 & -3.43 & -0.23 & -5.03 & 4.58 & 0.923 \\
\hline
\end{tabular}

${ }^{\mathrm{a}}$ Change $=$ month $12 T$-score minus month $6 T$-score

${ }^{\mathrm{b}} p$ values are unadjusted for multiple comparisons

influence of all five domains in HRQoL for participants with RYRI-RM. This slight difference highlights the value of the mixed methods approach because it allows for a more nuanced understanding of the data. Future thoughts and symptoms recorded lower percentage of comments than the remaining domains, suggesting that participants were more focused on current issues than upcoming problems, and that it was easier for them to accept physical limitations associated with their condition than social or psychological implications. A similar situation has previously supported this idea in individuals with ALS [51]. These results are similar to previous frequency analysis in NMD [24].

The analysis by visit displayed similar percentages for each domain, which is consistent with our findings for global analysis. Taking into account the number of quotes per visit, baseline interviews included more comments. This observation suggests that participants were inclined to provide more information at the beginning of the study as it was their first visit/interview. Perhaps this was also due to positive expectations of the trial [47]. Normalized percentages per visit reported similar results, revealing that participants provided comparable information in each visit. Finally, results for data transformation analysis by participant resulted in a similar trend to the global analysis findings.

As previously stated, $R Y R I$-RM are slowly progressive diseases, which makes it necessary to capture disease progression when considering effects of treatments and HRQoL. This issue was captured though the identification of the domain and subdomain of post-intervention and disease progression. Both concepts were analyzed in qualitative and data transformation analyses.

Three domains from Neuro-QoL showed some evidence of a difference between the NAC and placebo groups, fatigue, anxiety, and stigma. The $p$ values for these domains ranged between 0.014 and 0.034 , which do not reflect strong evidence of a difference given that 20 tests were performed. The sample size was small given the rare nature of the disease, and therefore the statistical power to detect differences was also small. Likewise, although there was a trend on the subjective self-assessment, suggesting a potential positive effect of NAC on participants with RYRI-RM, our sample 
size may be too small to produce strong evidence of a causal relationship between subjective self-assessment and NAC treatment. Thus, there was no clear evidence that any of the three tools captured positive changes as a result of NAC treatment on quantitative analysis. Interestingly, the results of our analyses of both subjective quantitative and qualitative/transformed data were similar to our findings from our primary endpoints in that NAC did not significantly improve any endpoints [9].

As mentioned, the main limitation in this study (for the transformed and quantitative analyses) was the sample size, which was small. Another major limitation was that all enrolled participants were ambulatory due to 6MWT being a primary endpoint, which meant that only mild-to-moderately affected individuals were enrolled.

\section{Conclusion}

Our qualitative analysis provides salient information on the major concerns/issues participants with RYRI-RM face and how they describe those concerns/issues affecting their HRQoL. We present a comprehensive characterization of HRQoL, in mild-to-moderately affected individuals, based on a symptom/patient-centered approach. These findings, especially the importance of social impacts, the development of several coping strategies, both physical and psychological, and the identification of fatigue and weakness as key symptoms, will inform future studies in this rare disease. However, at this dose, there was no clear evidence that NAC improved PRO scores for PROMIS or Neuro-QoL. Furthermore, although treatment benefits were mentioned by more (blinded) participants on NAC than placebo during qualitative interviews, once the qualitative data were transformed and quantified, evidence of a difference was not conclusive. Interestingly, these findings were similar to our primary endpoints and PRO results, in which 6MWT approached a clinically meaningful change, but no clear difference post-NAC treatment was identified in 6MWT or oxidative stress levels. Given the similar findings across our multiple methods and endpoints, the introduction of MMR, namely qualitative interviews and their data transformation, may be a valuable, complementary approach to clinical trials. Although this type of analysis requires a distinct skill set from many trial endpoints, MMR may be especially useful to incorporate in clinical studies in order to address and follow the FDA's guidance and prioritization on the inclusion of affected individuals' perspectives in clinical trials.

Acknowledgements The authors would like to thank all study participants for their sacrifice and commitment to be in this study. We also appreciate the support provided during the study by Ms. Karez Hawkins, Ms. Monique O. Shelton, and Dr. Joshua J. Todd (Neuromuscular
Symptoms Unit, National Institute of Nursing Research, National Institutes of Health). We would like to thank Dr. Paule V. Joseph (Sensory Science and Metabolism Unit, National Institute of Nursing Research, National Institutes of Health) and Dr. Joan K. Austin (Department of Environments for Health, School of Nursing, Indiana UniversityPurdue University Indianapolis) for reviewing the manuscript. Also, we acknowledge the RYR1 Foundation for assistance with recruitment for this study.

Author contributions Study conception and design: Dr. KGM; Acquisition of data: Ms. ICC, Ms. MSR, Mr. JSE, Ms. AK, and Dr. KGM; Analysis and interpretation of the data: Dr. CC-P, Ms. MMC, Ms. ICC, Dr. PGW, and Dr. KGM; Drafting the manuscript: Dr. CC-P, Ms. MMC, Dr. ME-B, Dr. PGW, and Dr. KGM; Revising the manuscript for intellectual content: Dr. CC-P, Ms. MMC, Ms. ICC, Dr. ME-B, Ms. MSR, Mr. JSE, Ms. AK, Dr. PGW, and Dr. KGM.

Funding This study was funded by the Intramural Programs of the National Institute of Nursing Research, National Institute of Neurological Disorders and Stroke, the National Institutes of Health Clinical Center, and Bench to Bedside Award (10-2013/Office of Rare Disease/ NINR).

\section{Compliance with ethical standards}

Conflict of interest Dr. Carlos Capella-Peris, Ms. Mary M. Cosgrove, Ms. Irene C. Chrismer, Dr. Magalie Emile-Backer, Ms. M. Sonia Razaqyar, Mr. Jeffrey S. Elliott, Ms. Anna Kuo, and Dr. Paul G. Wakim have no conflicts of interest. Dr. Katherine G. Meilleur has received support from the RYR-1 Foundation, and an NIH Clinical Center Bench to Bedside Award (10-2013/Office of Rare Disease/NINR).

\section{References}

1. U.S. Food and Drug Administration. (2009). Guidance for industry patient-reported outcome measures: Use in medical product development to support labeling claims. Rockville, MD: U.S. Food and Drug Administration. https://doi.org/10.11 11/j.1524-4733.2009.00609.x

2. U.S. Food and Drug Administration. (2018). Patient-focused drug development: Collecting comprehensive and representative input. Rockville, MD: U.S. Food and Drug Administration.

3. U.S. Food and Drug Administration. (2019). Patient-focused drug development: Methods to identify what is important to patients. Rockville, MD: U.S. Food and Drug Administration.

4. U.S. Food and Drug Administration. (2019). Rare diseases: Common issues in drug development. Guidance for industry. Rockville, MD: U.S. Food and Drug Administration. https://doi. org/10.1186/s13045-014-0070-8

5. Ferrans, C. E., \& Powers, M. J. (1985). Quality of life index: Development and psychometric properties. Advances in Nursing Science, 8(1), 15-24. https://doi.org/10.1097/00012272-19851 0000-00005.

6. Morel, T., \& Cano, S. J. (2017). Measuring what matters to rare disease patients-Reflections on the work by the IRDiRC taskforce on patient-centered outcome measures. Orphanet Journal of Rare Diseases, 12(1), 171. https://doi.org/10.1186/s1302 3-017-0718-x.

7. Amburgey, K., McNamara, N., Bennett, L. R., McCormick, M. E., Acsadi, G., \& Dowling, J. J. (2011). Prevalence of congenital myopathies in a representative pediatric united states population. 
Annals of Neurology, 70(4), 662-665. https://doi.org/10.1002/ ana.22510.

8. Todd, J. J., Razaqyar, M. S., Witherspoon, J. W., Lawal, T. A., Mankodi, A., Chrismer, I. C., et al. (2018). Novel variants in individuals with RYR1-related congenital myopathies: Genetic, laboratory, and clinical findings. Frontiers in Neurology, 9, 118. https://doi.org/10.3389/fneur.2018.00118.

9. Todd, J. J., Lawal, T. A., Witherspoon, J. W., Chrismer, I. C., Razaqyar, M. S., Punjabi, M., et al. (2019). A randomized controlled trial of $N$-acetylcysteine therapy for RYR1-related myopathies. Neurology, in press (under embargo).

10. Dlamini, N., Voermans, N. C., Lillis, S., Stewart, K., Kamsteeg, E.-J., Drost, G., et al. (2013). Mutations in RYR1 are a common cause of exertional myalgia and rhabdomyolysis. Neuromuscular Disorders, 23(7), 540-548. https://doi.org/10.1016/j. nmd.2013.03.008

11. Zhou, H., Jungbluth, H., Sewry, C. A., Feng, L., Bertini, E., Bushby, K., et al. (2007). Molecular mechanisms and phenotypic variation in RYR1-related congenital myopathies. Brain, 130(8), 2024-2036. https://doi.org/10.1093/brain/awm096.

12. van Ruitenbeek, E., Custers, J. A. E., Verhaak, C., Snoeck, M., Erasmus, C. E., Kamsteeg, E. J., et al. (2019). Functional impairments, fatigue and quality of life in RYR1-related myopathies: A questionnaire study. Neuromuscular Disorders, 29(1), 30-38. https://doi.org/10.1016/j.nmd.2018.10.006.

13. Dowling, J. J., Arbogast, S., Hur, J., Nelson, D. D., McEvoy, A., Waugh, T., et al. (2012). Oxidative stress and successful antioxidant treatment in models of RYR1-related myopathy. Brain, 135(4), 1115-1127. https://doi.org/10.1093/brain/aws036.

14. Durham, W. J., Aracena-Parks, P., Long, C., Rossi, A. E., Goonasekera, S. A., Boncompagni, S., et al. (2008). RyR1 S-nitrosylation underlies environmental heat stroke and sudden death in Y522S RyR1 knockin mice. Cell, 133(1), 53-65. https://doi.org/10.1016/j.cell.2008.02.042.

15. Cashion, A. K., \& Grady, P. A. (2015). The National Institutes of Health/National Institutes of Nursing Research intramural research program and the development of the National Institutes of Health Symptom Science Model. Nursing Outlook, 63(4), 484-487. https://doi.org/10.1016/j.outlook.2015.03.001.

16. Creswell, J. W., \& Plano Clark, V. L. (2007). Designing and conducting mixed methods research. Thousand Oaks, CA: Sage Publications.

17. Greene, J. C., Caracelli, V. J., \& Graham, W. F. (1989). Toward a conceptual framework for mixed-method evaluation designs. Educational Evaluation and Policy Analysis, 11(3), 255-274. https://doi.org/10.3102/01623737011003255.

18. Geirdal, A. Ø., Lund-Petersen, I., \& Heiberg, A. (2015). Understanding the experience of Myotonic Dystrophy. Mixed method study. Journal of Genetic Counseling, 24(1), 169-178. https:// doi.org/10.1007/s10897-014-9752-1.

19. Johnson, N. E., Luebbe, E., Eastwood, E., Chin, N., Moxley, R. T., \& Heatwole, C. R. (2014). The impact of congenital and childhood myotonic dystrophy on quality of life: A qualitative study of associated symptoms. Journal of Child Neurology, 29(7), 983-986. https://doi.org/10.1177/0883073813484804.

20. Vorster, N., Evans, K., Murphy, N., Kava, M., Cairns, A., Clarke, D., et al. (2019). Powered standing wheelchairs promote independence, health and community involvement in adolescents with Duchenne muscular dystrophy. Neuromuscular Disorders, 29(3), 221-230. https://doi.org/10.1016/j. nmd.2019.01.010.

21. Flick, U. (2014). An introduction to qualitative research (5th ed.). Thousand Oaks, CA: Sage Publications.

22. Saunders, B., Sim, J., Kingstone, T., Baker, S., Waterfield, J., Bartlam, B., et al. (2018). Saturation in qualitative research: Exploring its conceptualization and operationalization. Quality \& Quantity, 52(4), 1893-1907. https://doi.org/10.1007/s11135-017-0574-8.

23. LaFaver, K., Miyasaki, J. M., Keran, C. M., Rheaume, C., Gulya, L., Levin, K. H., et al. (2018). Age and sex differences in burnout, career satisfaction, and well-being in US neurologists. Neurology, 91(20), e1928-e1941. https://doi.org/10.1212/WNL.0000000000 006497.

24. Johnson, N. E., Quinn, C., Eastwood, E., Tawil, R., \& Heatwole, C. R. (2012). Patient-identified disease burden in facioscapulohumeral muscular dystrophy. Muscle \& Nerve, 46(6), 948-950. https://doi.org/10.1002/mus.23529.

25. Creswell, J. W. (2014). Research Design. Qualitative, quantitative and mixed methods approaches (4th ed.). London, UK: Sage Publications.

26. Miyasaki, J. M., Rheaume, C., Gulya, L., Ellenstein, A., Schwarz, H. B., Vidic, T. R., et al. (2017). Qualitative study of burnout, career satisfaction, and well-being among US neurologists in 2016. Neurology, 89(16), 1730-1738. https://doi.org/10.1212/ WNL.0000000000004526.

27. Cella, D., Riley, W., Stone, A., Rothrock, N., Reeve, B., Yount, S., et al. (2010). The Patient-Reported Outcomes Measurement Information System (PROMIS) developed and tested its first wave of adult self-reported health outcome item banks: 2005-2008. Journal of Clinical Epidemiology, 63(11), 1179-1194. https:// doi.org/10.1016/j.jclinepi.2010.04.011.

28. Cella, D., Nowinski, C., Peterman, A., Victorson, D., Miller, D., Lai, J.-S., et al. (2011). The neurology quality-of-life measurement initiative. Archives of Physical Medicine and Rehabilitation, 92(10), S28-S36. https://doi.org/10.1016/j.apmr.2011.01.025.

29. Abel, E. E. D. H., Cup, E. H. C., Lanser, A., Leclercq, W. K. G., Raaphorst, J., Padberg, G. W., et al. (2018). Experiences with bariatric surgery in patients with facioscapulohumeral dystrophy and myotonic dystrophy type 1: A qualitative study. Neuromuscular Disorders, 28(11), 938-946. https://doi.org/10.1016/j. nmd.2018.09.003.

30. Chen, Y. T., Shih, F. J., Hayter, M., Hou, C. C., \& Yeh, J. H. (2013). Experiences of living with myasthenia gravis: A qualitative study with taiwanese people. Journal of Neuroscience Nursing, 45(2), E3-E10. https://doi.org/10.1097/JNN.0b013e3182 $8291 \mathrm{a} 6$.

31. Davydov, D. M., Stewart, R., Ritchie, K., \& Chaudieu, I. (2010). Resilience and mental health. Clinical Psychology Review, 30(5), 479-495. https://doi.org/10.1016/j.cpr.2010.03.003.

32. Steptoe, A., Dockray, S., \& Wardle, J. (2009). Positive affect and psychobiological processes relevant to health. Journal of Personality, 77(6), 1747-1776. https://doi.org/10.111 1/j.1467-6494.2009.00599.x.

33. Jungbluth, H., Zhou, H., Hartley, L., Halliger-Keller, B., Messina, S., Longman, C., et al. (2005). Minicore myopathy with ophthalmoplegia caused by mutations in the ryanodine receptor type 1 gene. Neurology, 65(12), 1930-1935. https://doi.org/10.1212/01. wnl.0000188870.37076.f2.

34. Klein, A., Jungbluth, H., Clement, E., Lillis, S., Abbs, S., Munot, P., et al. (2011). Muscle magnetic resonance imaging in congenital myopathies due to ryanodine receptor type 1 gene mutations. Archives of Neurology, 68(9), 1171-1179. https://doi.org/10.1001/ archneurol.2011.188.

35. Hagerman, K. A., Howe, S. J., \& Heatwole, C. E. (2019). The myotonic dystrophy experience: A North American crosssectional study. Muscle \& Nerve, 59(4), 457-464. https://doi. org/10.1002/mus.26420.

36. Winter, Y., Schepelmann, K., Spottke, A. E., Claus, D., Grothe, C., Schröder, R., et al. (2010). Health-Related Quality of Life in ALS, myasthenia gravis and facioscapulohumeral muscular dystrophy. Journal of Neurology, 257(9), 1473-1481. https://doi. org/10.1007/s00415-010-5549-9. 
37. Bray, P., Bundy, A. C., Ryan, M. M., \& North, K. N. (2010). Feasibility of a computerized method to measure quality of "everyday" life in children with neuromuscular disorders. Physical \& Occupational Therapy In Pediatrics, 30(1), 43-53. https://doi. org/10.3109/01942630903294687.

38. Miladi, N., Bourguignon, J.-P., \& Hentati, F. (1999). Cognitive and psychological profile of a Tunisian population of limb girdle muscular dystrophy. Neuromuscular Disorders, 9(5), 352-354. https://doi.org/10.1016/S0960-8966(99)00006-1.

39. Karimi, M., \& Brazier, J. (2016). Health, Health-Related Quality of Life, and quality of life: What is the difference? PharmacoEconomics, 34(7), 645-649. https://doi.org/10.1007/s4027 3-016-0389-9.

40. Witting, N., Werlauff, U., Duno, M., \& Vissing, J. (2017). Phenotypes, genotypes, and prevalence of congenital myopathies older than 5 years in Denmark. Neurology Genetics, 3(2), e140. https:// doi.org/10.1212/NXG.0000000000000140.

41. Dowling, J. J., Lillis, S., Amburgey, K., Zhou, H., Al-Sarraj, S., Buk, S. J. A., et al. (2011). King-Denborough syndrome with and without mutations in the skeletal muscle ryanodine receptor (RYR1) gene. Neuromuscular Disorders, 21(6), 420-427. https:// doi.org/10.1016/j.nmd.2011.03.006.

42. Jungbluth, H., Müller, C. R., Halliger-Keller, B., Brockington, M., Brown, S. C., Feng, L., et al. (2002). Autosomal recessive inheritance of RYR1 mutations in a congenital myopathy with cores. Neurology, 59(2), 284-287. https://doi.org/10.1212/ WNL.59.2.284.

43. Matthews, E., Neuwirth, C., Jaffer, F., Scalco, R. S., Fialho, D., Parton, M., et al. (2018). Atypical periodic paralysis and myalgia. Neurology, 90(5), e412-e418. https://doi.org/10.1212/ WNL.0000000000004894.

44. Scacheri, P. C., Hoffman, E. P., Fratkin, J. D., Semino-Mora, C., Senchak, A., Davis, M. R., et al. (2000). A novel ryanodine receptor gene mutation causing both cores and rods in congenital myopathy. Neurology, 55(11), 1689-1696. https://doi.org/10.1212/ WNL.55.11.1689.

45. Capacchione, J. F., Sambuughin, N., Bina, S., Mulligan, L. P., Lawson, T. D., \& Muldoon, S. M. (2010). Exertional rhabdomyolysis and malignant hyperthermia in a patient with ryanodine receptor type 1 gene, L-type calcium channel $\alpha-1$ subunit gene, and calsequestrin-1 gene polymorphisms. Anesthesiology, 112(1), 239-244. https://doi.org/10.1097/ALN.0b013e3181c29504.

46. Davis, M., Brown, R., Dickson, A., Horton, H., James, D., Laing, N., et al. (2002). Malignant hyperthermia associated with exercise-induced rhabdomyolysis or congenital abnormalities and a novel RYR1 mutation in New Zealand and Australian pedigrees. British Journal of Anaesthesia, 88(4), 508-515. https://doi. org/10.1093/bja/88.4.508.

47. Bingel, U., Wanigasekera, V., Wiech, K., Ni Mhuircheartaigh, R., Lee, M. C., Ploner, M., et al. (2011). The effect of treatment expectation on drug efficacy: Imaging the analgesic benefit of the opioid Remifentanil. Science Translational Medicine, 3(70), 70ra14. https://doi.org/10.1126/scitranslmed.3001244.

48. Albrecht, G. L., \& Devlieger, P. J. (1999). The disability paradox: High quality of life against all odds. Social Science \& Medicine, 48(8), 977-988. https://doi.org/10.1016/S0277-9536(98)00411-0.

49. de Oliveira, C. M., \& de Araújo, A. P. Q. C. (2011). Self-reported quality of life has no correlation with functional status in children and adolescents with spinal muscular atrophy. European Journal of Paediatric Neurology, 15(1), 36-39. https://doi.org/10.1016/j. ejpn.2010.07.003.

50. Landfeldt, E., Lindgren, P., Bell, C. F., Guglieri, M., Straub, V., Lochmüller, H., et al. (2016). Health-Related Quality of Life in patients with Duchenne muscular dystrophy: A multinational, cross-sectional study. Developmental Medicine \& Child Neurology, 58(5), 508-515. https://doi.org/10.1111/dmcn.12938.

51. Simmons, Z., Bremer, B. A., Robbins, R. A., Walsh, S. M., \& Fischer, S. (2000). Quality of life in ALS depends on factors other than strength and physical function. Neurology, 55(3), 388-392. https://doi.org/10.1212/WNL.55.3.388.

Publisher's Note Springer Nature remains neutral with regard to jurisdictional claims in published maps and institutional affiliations. 